\title{
On Parallel Machine Replacement Problems with General Replacement Cost Functions and Stochastic Deterioration
}

\author{
Suzanne Childress, ${ }^{1}$ Pablo Durango-Cohen ${ }^{2}$ \\ ${ }^{1}$ Department of Industrial Engineering and Management Sciences, Northwestern University, \\ 2145 Sheridan Road, Evanston, Illinois 60208 \\ ${ }^{2}$ Department of Civil and Environmental Engineering-Transportation Center, Northwestern University, \\ 2145 Sheridan Road, A335, Evanston, Illinois 60208
}

Received 18 December 2003; revised 7 March 2005; accepted 24 March 2005

DOI 10.1002/nav.20088

Published online 28 April 2005 in Wiley InterScience (www.interscience.wiley.com).

\begin{abstract}
The parallel machine replacement problem consists of finding a minimum cost replacement policy for a finite population of economically interdependent machines. In this paper, we formulate a stochastic version of the problem and analyze the structure of optimal policies under general classes of replacement cost functions. We prove that for problems with arbitrary cost functions, there can be optimal policies where a machine is replaced only if all machines in worse states are replaced (Worse Cluster Replacement Rule). We then show that, for problems with replacement cost functions exhibiting nonincreasing marginal costs, there are optimal policies such that, in any stage, machines in the same state are either all kept or all replaced (No-Splitting Rule). We also present an example that shows that economies of scale in replacement costs do not guarantee optimal policies that satisfy the No-Splitting Rule. These results lead to the fundamental insight that replacement decisions are driven by marginal costs, and not by economies of scale as suggested in the literature. Finally, we describe how the optimal policy structure, i.e., the No-Splitting and Worse Cluster Replacement Rules, can be used to reduce the computational effort required to obtain optimal replacement policies. (c) 2005 Wiley Periodicals, Inc. Naval Research Logistics 52: 409-419, 2005.
\end{abstract}

Keywords: equipment replacement; economic interactions; dynamic programming; Markov decision processes

\section{INTRODUCTION}

The parallel machine replacement problem (PMRP) consists of finding a minimum cost replacement policy for a finite population of economically interdependent machines. At the start of each decision-making stage, one may choose to either keep a machine or to replace it with a new one. The economic interactions considered in the literature are related to budget constraints that limit the number of machines that can be replaced in each stage $[8,5]$; constraints that limit the number of machines that can be replaced in each stage in order to guarantee enough capacity to satisfy demand [12]; and the structure of the replacement cost function [7]. This paper addresses the latter issue, a PMRP where replacement

Correspondence to: P.L. Durango-Cohen (pdc@northwestern. edu); S. Childress (s-childress@ northwestern.edu) decisions for individual machines are linked by the replacement cost structure.

We build on the model introduced by Jones, Zydiak, and Hopp [7] in which machines deteriorate deterministically over time. Replacement decisions for the individual machines are linked by a fixed cost incurred whenever one or more of the machines are replaced. Variable costs per unit are also incurred for the replacement, maintenance, operation, and salvage of machines. Replacement decisions trade off replacement costs with nondecreasing maintenance and operating costs, and with salvage values that are nonincreasing with machine age. The authors present two intuitive rules that limit the number of options that need to be considered in each stage, and therefore, reduce the complexity of finding optimal replacement policies. The No-Splitting Rule (NSR) ensures that there is an optimal replacement policy such that in any stage machines of the same age are either 
all kept or all replaced. The Older Cluster Replacement Rule (OCRR) guarantees that there is an optimal policy such that a machine is replaced only if all older machines are replaced.

In this paper, we present a generalization of the problem which we term the stochastic PMRP. The stochastic PMRP consists of a set of independent Markovian processes, with each process corresponding to the deterioration of one of the machines that comprises the population. We also consider general classes of replacement cost functions instead of the fixed plus per-unit variable cost structure used in the literature.

In addition to presenting a formulation for the stochastic PMRP, we provide structural and computational insights about the problem. We show that, under the assumption of an increasing failure rate, the structure of optimal replacement policies for the deterministic PMRP extends to the stochastic version of the problem. Specifically, we prove that optimal policies for the stochastic PMRP with arbitrary replacement cost functions satisfy a result analogous to the OCRR. We then extend the NSR for replacement functions with nonincreasing marginal costs. We also present an example that shows that economies of scale in replacement costs do not guarantee optimal replacement policies that satisfy the NSR. Together, these results lead to the fundamental insight that replacement decisions are driven by marginal costs, and not by economies of scale as is suggested in the PMRP literature. Finally, we illustrate how the structural results can be used to reduce the computational effort required to find optimal replacement policies.

The remainder of the paper is organized as follows. In Section 2, we provide an overview of the deterministic PMRP and the assumptions used in the literature for its analysis. We also summarize the existing structural results for the problem. We formulate the stochastic PMRP and characterize classes of optimal replacement policies in Section 3. In Section 4, we describe how the structure of optimal policies can be used to reduce the computational effort to solve the problem. We conclude in Section 5 by summarizing the contributions of the paper.

\section{LITERATURE REVIEW}

The deterministic PMRP introduced by Jones, Zydiak, and Hopp [7] consists of finding a minimum cost replacement policy for a finite population of machines over a planning horizon that can be finite or infinite. The authors define a group of machines of the same age to be a cluster. We denote the size of the population and the initial number of clusters $N$ and $n$, respectively. At the start of each stage, the decisions are to either keep a machine or to replace it with a new one. Thus, one must consider a total of $2^{N}$ replacement options in each stage. In the case of a $T$-stage problem, all machines are salvaged at the end of the planning horizon (start of stage $T+1$ ).

Prior to introducing notation to describe the deterministic PMRP, we note that the structural properties and managerial insights developed in the literature are for the general case of time-varying parameters, i.e., the parameters and functions comprising the models in the literature are indexed with a subscript $t$ that represents the stage for which they apply. To simplify the notation in the paper, we focus on the case of stationary parameters. However, and unless noted otherwise, the proofs and discussion also apply to the case of time-varying parameters. Hence, the models in the deterministic PMRP literature can be specified with the following parameters and functions:

$m(i)$ : maintenance and operating costs of an $i$-year old machine;

$s(i): \quad$ salvage value of an $i$-year old machine;

$p: \quad$ variable purchase cost of a new machine;

$K$ : fixed cost incurred if any purchases occur in a given stage; and

$\delta$ : discount factor $[\delta=1 /(1+r)$ where $r$ is the discount rate associated with the time value of money].

McClurg and Chand [9] define the function $O(i) \equiv$ $m(i)+s(i)-\delta s(i+1)$ to account for both the maintenance and operating costs of an $i$-year old machine, as well as its depreciation in a given stage. With the parameters introduced above, the replacement cost function, $R(y)$, defined as the cost of replacing $y$ machines in a given stage, has the form:

$$
R(y)= \begin{cases}0, & y=0, \\ K+p \cdot y, & y>0 .\end{cases}
$$

Jones, Zydiak, and Hopp [7] show that the replacement cost structure in (1) leads to the NSR. The NSR guarantees that there is an optimal replacement policy such that in any stage machines of the same age are either all kept or all replaced. The NSR, furthermore, ensures that machines remain in the same cluster through the end of the planning horizon. Consequently, the state of the system can be defined as the sizes and ages of the clusters that comprise the population. This results in a decrease in the number of replacement options that need to be considered in each stage from $2^{N}$ to at most $2^{n}$.

In addition to the NSR, optimal policies for the PMRP can satisfy other properties that depend on parameter as- 
sumptions. Next, we present the different assumptions considered in the literature, followed by a summary of the structural results.

a. For $i \geq 0, m(i)$ is nondecreasing in $i$, i.e., for $j \geq i$, $m(j) \geq m(i)$.

b. For $i \geq 0, s(i)$ is nonincreasing in $i$, i.e., for $j \geq i$, $s(j) \leq s(i)$; and $s(0)=p$.

c. For $i \geq 0, m(i)+s(i)$ is nondecreasing in $i$, i.e., for $j \geq i, m(j)+s(j) \geq m(i)+s(i)$.

$\hat{c}$. For $i \geq 1, m(i)+s(i)$ is nondecreasing in $i$, i.e., for $j \geq i \geq 1, m(j)+s(j) \geq m(i)+s(i)$.

d. For $i \geq 0, m(i)+s(i) \geq m(0)+s(0)$.

e. For $i \geq 1, O(i+1) \geq O(i)$.

An additional assumption from the deterministic PMRP literature is that machines of a given maximum allowable age denoted $\bar{a}$ must be replaced.

Jones, Zydiak, and Hopp [7] show that for the replacement cost function given in Eq. (1), assumptions (a), (b), and (c) lead to the OCRR; that is, there is an optimal policy such that a machine is replaced only if all older machines are replaced. Tang and Tang [10] make use of the assumption that $s(0)=p$, and replace assumption (c) with (d) (a more general assumption) in order to establish that there is an optimal policy that satisfies a stronger result, the All Or None Rule (AONR). The AONR states there is an optimal policy such that in each stage machines in the population are either all kept or all replaced. Hopp, Jones, and Zydiak [6] argue that assumption (d) places an undue restriction on the purchase price of a new machine. Furthermore, they show that if assumption (c) only applies to $i \geq 1$, then the AONR does not hold but the OCRR does. This modification corresponds to assumption $(\hat{c})$, which we use in the remainder of the paper. Finally, McClurg and Chand [9] replace assumption $(\hat{c})$ with (e), which they claim is more commonly observed in practice due to the high decline in salvage values in the early stages of operation of a machine. They find that, for the replacement cost function given in Eq. (1) and under assumptions (a), (b), and (e), there is an optimal policy that satisfies the OCRR.

\section{THE STOCHASTIC PARALLEL MACHINE REPLACEMENT PROBLEM}

In this section, we introduce the stochastic PMRP and show that, under the assumption of an increasing failure rate, the structure of optimal replacement policies for the deterministic PMRP extends to the stochastic version of the problem. Specifically, we prove that the assumptions used to establish the NSR and the OCRR for the deterministic
PMRP are sufficient to extend the rules to the stochastic PMRP with increasing failure rate.

\subsection{Model Formulation}

We formulate the stochastic PMRP as a set of $N$ independent Markovian processes, with each process corresponding to the deterioration of one of the $N$ machines that comprise the population. The costs in the model are the same as in the deterministic version of the problem, and thus, the replacement cost function, $R(y)$, links the replacement decisions. Each machine deterioration process is defined over a finite set of states, $\mathscr{Y}=\{0,1,2, \ldots, \bar{a}\}$, whose elements are ordered from best/new to worst/failed. We also assume that all costs in the model are bounded. ${ }^{1}$ The Markovian deterioration processes require the specification of a set of probabilities, $p_{i j}, \forall i, j \in \mathscr{Y}$, where $p_{i j}$ is the probability that a machine transitions from state $i$ to state $j$ in a stage when the decision is to keep it. Because replacements occur at the start of a stage, $p_{0 j}$ is the probability that a newly-replaced machine transitions into state $j$.

We assume that machines exhibit an increasing failure rate as defined by Derman [3]. The increasing failure rate assumption states that machines in better states are more likely to remain in better states than machines in worse states. Mathematically, the assumption is expressed as

$$
\sum_{j=0}^{l} p_{i j} \geq \sum_{j=0}^{l} p_{k j}, \quad \forall l, \quad i \leq k \in \mathscr{Y} .
$$

Derman [3] shows that for every nondecreasing function $g(\cdot)$, expression (2) is equivalent to

$$
\sum_{j \in \mathscr{S}} p_{i j} g(j) \leq \sum_{j \in \mathscr{S}} p_{k j} g(j), \quad \forall i \leq k \in \mathscr{Y} .
$$

Hereafter, we refer to this result as Derman's Lemma.

We now present a dynamic programming formulation for the stochastic PMRP that is similar to the one for the deterministic PMRP in [7]. We represent the state of the system in stage $t$ of a stochastic PMRP as an $N$-dimensional vector, $\vec{X}^{t}=$ $\left[X_{1}^{t}, X_{2}^{t}, \ldots, X_{N}^{t}\right]$, in which each vector component corresponds to the state of a machine. Recall that a policy is a mapping that specifies a decision for each machine, for every possible stage and state combination. We denote the $N$-dimensional mapping for stage $t$ and state $\vec{X}^{t}, \vec{\mu}\left(t, \vec{X}^{t}\right)$, in which each component $\mu_{i}(t$, $\left.\vec{X}^{\natural}\right)$ is defined as follows:

\footnotetext{
${ }^{1}$ These assumptions are consistent with the deterministic PMRP and ensure that the expectations in the remainder of the paper are well defined. The assumptions also guarantee the existence of an optimal replacement policy.
} 


$$
\mu_{i}\left(t, \vec{X}^{t}\right)= \begin{cases}1, & \text { when policy } \mu \text { specifies that for stage } t \text { and state } \vec{X}^{t} \\ \text { machine } i \text { is to be replaced with a new one } & \text { otherwise }\end{cases}
$$

The expected discounted cost of managing the system in state $\vec{X}^{t}$ under policy $\mu$ from the start of stage $t$ until the end of the planning horizon, given the state of the system, is written recursively as follows:

$$
\begin{aligned}
& f_{t}^{\mu}\left(\vec{X}^{t}\right)=R\left(\sum_{i=1}^{N} \mu_{i}\left(t, \vec{X}^{t}\right)\right)+\sum_{i=1}^{N} \mu_{i}\left(t, \vec{X}^{t}\right)\left[-s\left(X_{i}^{t}\right)+m(0)\right] \\
& +\sum_{i=1}^{N}\left(1-\mu_{i}\left(t, \vec{X}^{t}\right)\right) m\left(X_{i}^{t}\right)+\delta \cdot \mathbf{E}\left[f_{t+1}^{u}\left(\vec{X}^{t+1}\right) \mid \vec{X}^{t}, \vec{\mu}\left(t, \vec{X}^{t}\right)\right] .
\end{aligned}
$$

The first three terms correspond to the costs incurred in stage $t$ and the last term is for the sum of discounted expected costs from the start of $t+1$ until the end of the planning horizon. In the case of a finite horizon problem, all machines are salvaged at the start of stage $T+1$. Thus, the boundary condition for a given state, $\vec{X}^{T+1}$, and policy, $\mu$, are given by the following expression:

$$
f_{T+1}^{\mu}\left(\vec{X}^{T+1}\right)=-\sum_{i=1}^{N} s\left(X_{i}^{T+1}\right) .
$$

The optimal objective value function for each stage and state is defined as the minimum expected discounted cost of managing the system from the given stage and state through the end of the planning horizon. The optimal objective value function is given by a replacement policy that minimizes the right-hand side of Eqs. (5) and (6) for every stage and state. In the remainder of the paper, we use $\mu^{*}$ to denote such optimal policies.

\subsection{The Worse Cluster Replacement Rule}

In this section we begin to characterize optimal replacement policies for the stochastic PMRP. We show that the assumptions used to establish the OCRR for the determin- istic PMRP guarantee the existence of optimal replacement policies with the same structure for the stochastic PMRP with increasing failure rate. We refer to the result as the Worse Cluster Replacement Rule (WCRR) because in the stochastic version of the problem machine condition does not correspond to age. First, we present an intermediate lemma:

LEMMA 1: Under assumptions (a) and (b), the optimal objective value function of the stochastic PMRP with increasing failure rate is nondecreasing in each component of the state-space, i.e.,

$$
\begin{gathered}
f_{t}^{\mu^{*}}\left(i, X_{2}^{t}, \ldots, X_{N}^{t}\right) \leq f_{t}^{\mu^{*}}\left(j, X_{2}^{t}, \ldots, X_{N}^{t}\right), \quad \forall i \leq j \in \mathscr{Y}, \\
X_{2}^{t}, \ldots, X_{N}^{t} \in \mathscr{G}^{N-1}, \quad t=1,2, \ldots, T+1 .
\end{gathered}
$$

PROOF: See the Appendix.

Lemma 1 states that the optimal expected discounted costs associated with machines in better states are no greater than those with machines in worse states. Next, we present theorems that describe the structure of replacement policies for the stochastic PMRP with increasing failure rate.

THEOREM 1 (The Worse Cluster Replacement Rule): Under assumptions (a), (b), and ( $\hat{c})$, the stochastic PMRP with increasing failure rate has an optimal policy such that a machine is replaced only if all machines in worse states are replaced.

PROOF: We prove the result by contradiction. Suppose there exists an instance of the stochastic PMRP where there is no optimal policy satisfying the WCRR. Let $\hat{\mu}$ be an optimal policy for the given instance. Without loss of generality, there is a stage $t$ and state $i, j, X_{3}^{t}, \ldots, X_{N}^{t}$ where $\hat{\mu}$ specifies that machine 1 in state $i$ is to be replaced and machine 2 in state $j$ is to be kept $(0<i<j)$. We also consider a policy $\bar{\mu}$, which differs from $\hat{\mu}$ in that the machine in state $i$ is kept and the machine in state $j$ is replaced in stage $t$. The policies are otherwise identical. We have that

$f_{t}^{\hat{u}}\left(i, j, X_{3}^{t}, \ldots, X_{N}^{t}\right)-f_{t}^{\bar{u}}\left(i, j, X_{3}^{t}, \ldots, X_{N}^{t}\right)=\underbrace{m(j)-m(i)+s(j)-s(i)}$

$$
+\delta \cdot \underbrace{\left\{\begin{array}{c}
\mathbf{E}\left[f_{t+1}^{\hat{\imath}}\left(\vec{X}^{t+1} \mid i, j, X_{3}^{t}, \ldots, X_{N}^{t}, \hat{\mu}\left(t, i, j, X_{3}^{t}, \ldots, X_{N}^{t}\right)\right)\right] \\
-\mathbf{E}\left[f_{t+1}^{\bar{t}}\left(\vec{X}^{t+1} \mid i, j, X_{3}^{t}, \ldots, X_{N}^{t}, \bar{\mu}\left(t, i, j, X_{3}^{t}, \ldots, x_{*}^{t}\right)\right)\right]
\end{array}\right\}}_{(* *)} .
$$


Assumption ( $\hat{c})$ implies that term (*) is nonnegative. The nonnegativity of term (**) follows from Lemma 1 and Derman's Lemma. The expectations are evaluated by conditioning on the realizations of $X_{3}^{t+1}, \ldots, X_{N}^{t+1}$ as is done in the proof of Lemma 1. Consequently, $f_{t}^{\hat{\mu}}\left(i, j, X_{3}^{t}, \ldots, X_{N}^{t}\right)-f_{t}^{\bar{\mu}}\left(i, j, X_{3}^{t}, \ldots\right.$, $\left.X_{N}^{t}\right) \geq 0$. The same argument can be repeated to construct a policy that satisfies the WCRR and that is at least as good as policy $\hat{\mu}$. This contradicts the assumption that there is no optimal policy satisfying the WCRR and completes the proof.

We extend Theorem 1 to cases where assumption $(\hat{c})$ is replaced with assumption (e). In other words, we extend the OCRR, under the assumptions of McClurg and Chand [9], to the stochastic PMRP with increasing failure rate. The extension requires modifying the cost function $O(\cdot)$, as follows, so that it lends itself to the analysis of stochastic deterioration:

$$
O(i) \equiv \begin{cases}m(0)-\delta \sum_{j \in \mathscr{S}} p_{0 j} s(j), & i=0, \\ m(i)+s(i)-\delta \sum_{j \in \mathscr{S}} p_{i j} s(j), & i \geq 1 .\end{cases}
$$

In addition, we assume that assumption (e) applies to the above cost function. These modifications constitute generalizations of the original cost function and assumption because the deterministic PMRP can be obtained as an instance of the stochastic PMRP defined over the set of states $\{0,1,2, \ldots, \bar{a}\}$ with the following transition probabilities: $p_{i j}=\{1$ for $j=i+1 ; 0$ otherwise $\}$, if a machine is kept; and $p_{i j}=\{1$ for $j=1 ; 0$ otherwise \}, if a machine is replaced with a new one.
THEOREM 2: Under assumptions (a), (b), and (e), the stochastic PMRP with increasing failure rate has an optimal policy that satisfies the WCRR, i.e., a policy where a machine is replaced only if all machines in worse states are replaced.

PROOF: We begin by defining an auxiliary function, $h_{t}^{\mu}\left(\vec{X}^{t}\right)$, as the expected discounted (modified) cost of managing a system in state $\vec{X}^{t}$ under policy $\mu$ from the start of stage $t$ until the end of the planning horizon. The function can be written recursively as follows:

$$
\begin{gathered}
h_{t}^{\mu}\left(\vec{X}^{t}\right)=R\left(\sum_{i=1}^{N} \mu_{i}\left(t, \vec{X}^{t}\right)\right)+\sum_{i=1}^{N} \mu_{i}\left(t, \vec{X}^{t}\right) O(0)+\sum_{i=1}^{N}(1 \\
\left.-\mu_{i}\left(t, \vec{X}^{t}\right)\right) O\left(X_{i}^{t}\right)+\delta \cdot \mathbf{E}\left[h_{t+1}^{\mu}\left(\vec{X}^{t+1}\right) \mid \vec{X}^{t}, \vec{\mu}\left(t, \vec{X}^{t}\right)\right] .
\end{gathered}
$$

In the case of a finite horizon problem, we assume that all machines are salvaged at the start of stage $T+1$. Thus, the boundary condition for a given state $\vec{X}^{T+1}$ and policy $\mu$ are given by the following expression:

$$
\begin{aligned}
& h_{T}^{\mu}\left(\vec{X}^{T}\right)=R\left(\sum_{i=1}^{N} \mu_{i}(T,\right.\left.\left.\vec{X}^{T}\right)\right) \\
&+\sum_{i=1}^{N} \mu_{i}\left(T, \vec{X}^{T}\right) O(0) \\
&+\sum_{i=1}^{N}\left(1-\mu_{i}\left(T, \vec{X}^{T}\right)\right) O\left(X_{i}^{T}\right) .
\end{aligned}
$$

We show by induction that $f_{t}^{\mu}\left(\vec{X}^{t}\right)=h_{t}^{\mu}\left(\vec{X}^{t}\right)-\sum_{i=1}^{N} s\left(\vec{X}_{i}^{t}\right)$. The result is immediate for stage $T$. For an arbitrary stage $t$ given that the result holds for stage $t+1$, we have

$$
\begin{aligned}
h_{t}^{\mu}\left(\vec{X}^{t}\right)-\sum_{i=1}^{N} s\left(\vec{X}_{i}^{t}\right)= & R\left(\sum_{i=1}^{N} \mu_{i}\left(t, \vec{X}^{t}\right)\right)+\sum_{i=1}^{N} \mu_{i}\left(t, \vec{X}^{t}\right) O(0) \\
& +\sum_{i=1}^{N}\left(1-\mu_{i}\left(t, \vec{X}^{t}\right)\right) O\left(X_{i}^{t}\right)+\delta \cdot \mathbf{E}\left[h_{t+1}^{\mu}\left(\vec{X}^{t+1}\right) \mid \vec{X}^{t}, \vec{\mu}\left(t, \vec{X}^{t}\right)\right]-\sum_{i=1}^{N} s\left(\vec{X}_{i}^{t}\right) \\
= & R\left(\sum_{i=1}^{N} \mu_{i}\left(t, \vec{X}^{t}\right)\right)+\sum_{i=1}^{N} \mu_{i}\left(t, \vec{X}^{t}\right)\left[-s\left(X_{i}^{t}\right)+m(0)\right] \\
& +\sum_{i=1}^{N}\left(1-\mu_{i}\left(t, \vec{X}^{t}\right)\right) m\left(X_{i}^{t}\right)+\delta \cdot \mathbf{E}\left[h_{t+1}^{\mu}\left(\vec{X}^{t+1}\right)-\sum_{i=1}^{N} s\left(\vec{X}_{i}^{t+1}\right) \mid \vec{X}^{t}, \vec{\mu}\left(t, \vec{X}^{t}\right)\right] \\
= & R\left(\sum_{i=1}^{N} \mu_{i}\left(t, \vec{X}^{t}\right)\right)+\sum_{i=1}^{N} \mu_{i}\left(t, \vec{X}^{t}\right)\left[-s\left(X_{i}^{t}\right)+m(0)\right]+\sum_{i=1}^{N}\left(1-\mu_{i}\left(t, \vec{X}^{t}\right)\right) m\left(X_{i}^{t}\right) \\
+ & \delta \cdot \mathbf{E}\left[f_{t+1}^{\mu}\left(\vec{X}^{t+1}\right) \mid \vec{X}^{t}, \vec{\mu}\left(t, \vec{X}^{t}\right)\right] \text { by the induction hypothesis. }
\end{aligned}
$$


We see that the last expression is equal to $f_{t}^{\mu}\left(\vec{X}^{t}\right)$. The problems of finding policies that minimize $f_{t}^{\mu}\left(\vec{X}^{t}\right)$ and $h_{t}^{\mu}\left(\vec{X}^{t}\right)$ for each stage and state are equivalent because the difference in the two value functions is a sunk cost, i.e., a cost that is independent of the set of decisions for each stage and state.

To show that assumption (e) guarantees that there is an optimal replacement policy that satisfies the WCRR for the stochastic PMRP with increasing failure rate, we note that the problem of finding a policy that minimizes $h_{t}^{\mu}\left(\vec{X}^{t}\right)$ can be solved by minimizing $f_{t}^{\mu}\left(\vec{X}^{t}\right)$ with $s(i)=0$ and $m(i)=$ $O(i), \forall i$. Assumption (e) for the problem of minimizing $h_{t}^{\mu}\left(\vec{X}^{t}\right)$ ensures that the new problem satisfies assumptions (a), (b), and $(\hat{c})$. Therefore, by Theorem 1 there is an optimal policy that satisfies the WCRR.

The results in this section do not depend on the structure of the replacement cost function which means that the WCRR is valid for deterministic and stochastic PMRPs with arbitrary replacement cost functions. Our proofs differ from earlier proofs of the OCRR for the deterministic PMRP that rely on a function consisting of fixed plus variable costs.

\subsection{The No-Splitting Rule}

In the previous subsection, we established that the WCRR does not depend on the structure of the replacement cost function. In this section, we show, on the other hand, that the NSR does depend on the structure of the replacement cost function. Jones, Zydiak, and Hopp [7] showed that fixed plus variable replacement costs guarantee optimal replacement policies satisfying the NSR. Here, we extend the NSR to the stochastic PMRP with increasing failure rate for replacement cost functions with nonincreasing marginal costs. We begin by defining functions with nonincreasing marginal costs (cf. [11]).

DEFINITION 1: A replacement cost function, $R(y)$, exhibits nonincreasing marginal costs if

$$
\begin{aligned}
& R\left(y_{2}+\Delta\right)-R\left(y_{2}\right) \leq R\left(y_{1}+\Delta\right)-R\left(y_{1}\right), \\
& \forall \Delta \geq 0, \quad y_{2} \geq y_{1} \geq 0 .
\end{aligned}
$$

The following theorem proves that the NSR holds for the stochastic PMRP with increasing failure rate and nonincreasing marginal costs.

THEOREM 3 (The No-Splitting Rule): Replacement cost functions with nonincreasing marginal costs guarantee that there is an optimal policy that satisfies the
NSR for the stochastic PMRP with increasing failure rate. That is, there is an optimal policy such that in any stage machines in the same state are either all kept or all replaced.

PROOF: We proceed by contradiction. Suppose that there is an instance of the stochastic PMRP with increasing failure rate and a replacement cost function with nonincreasing marginal costs such that there is no optimal policy satisfying the NSR. Let $\bar{\mu}$ be an optimal policy for the problem instance. This means that there is a stage $\bar{t}$ and a state $\bar{X}^{\bar{t}}$, where $\bar{\mu}$ specifies different actions for two machines that are part of a cluster in state $k$. Let the indices 1 and 2 correspond to two machines in a state $k$ with $\bar{\mu}_{1}=1$ and $\bar{\mu}_{2}=0$.

The optimality of $\bar{\mu}$ implies that the incremental value of replacing machine 1 instead of keeping it is nonnegative. That is,

$$
\begin{array}{r}
R\left(\left[\sum_{i=1}^{N} \bar{\mu}_{i}\left(\bar{t}, \bar{X}^{\bar{t}}\right)\right]-1\right)-R\left(\sum_{i=1}^{N} \bar{\mu}_{i}\left(\bar{t}, \bar{X}^{\bar{t}}\right)\right)+m(k) \\
+s(k)-m(0) \\
+\delta \cdot\left[\mathbf{E}\left[f_{\bar{t}+1}^{\bar{\mu}}\left(\bar{X}^{\bar{t}+1}\right) \mid \bar{X}^{\bar{t}}, 0,0, \bar{\mu}_{3}\left(\bar{t}, \bar{X}^{\bar{t}}\right), \ldots, \bar{\mu}_{N}\left(\bar{t}, \bar{X}^{\dagger}\right)\right]\right. \\
\left.-\mathbf{E}\left[f_{\bar{t}+1}^{\bar{\mu}}\left(X^{\bar{t}+1}\right) \mid \bar{X}^{\bar{t}}, \bar{\mu}\left(\bar{t}, \bar{X}^{\bar{t}}\right)\right]\right] \geq 0 .
\end{array}
$$

Similarly, the incremental value of replacing both machines together for $\bar{t}$ and $\bar{X}^{\bar{t}}$ instead of splitting them is

$$
\begin{aligned}
& R\left(\sum_{i=1}^{N} \bar{\mu}_{i}\left(\bar{t}, \bar{X}^{\dagger}\right)\right)-R\left(\left[\sum_{i=1}^{N} \bar{\mu}_{i}\left(\bar{t}, \bar{X}^{\dagger}\right)\right]+1\right)+m(k)+s(k) \\
& -m(0)+\delta \cdot\left[\mathbf{E}\left[f_{\bar{t}+1}^{\bar{\mu}}\left(\bar{X}^{i+1}\right) \mid \overline{X^{t}}, \bar{\mu}\left(\bar{t}, \bar{X}^{\bar{\dagger}}\right)\right]\right. \\
& \left.-\mathbf{E}\left[f_{\bar{t}+1}^{\bar{\mu}}\left(\bar{X}^{\overline{t+1}}\right) \mid \bar{X}^{t}, 1,1, \bar{\mu}_{3}\left(\bar{t}, \bar{X}^{\bar{X}}\right), \ldots, \bar{\mu}_{N}\left(\bar{t}, \bar{X}^{\dagger}\right)\right]\right] .
\end{aligned}
$$

Since $R(y)$ has nonincreasing marginal costs,

$$
\begin{aligned}
& R\left(\sum_{i=1}^{N} \bar{\mu}_{i}\left(\bar{t}, \bar{X}^{\dagger}\right)\right)-R\left(\left[\sum_{i=1}^{N} \bar{\mu}_{i}\left(\bar{t}, \bar{X}^{\dagger}\right)\right]+1\right) \\
& \quad \geq R\left(\left[\sum_{i=1}^{N} \bar{\mu}_{i}\left(\bar{t}, \bar{X}^{\dagger}\right)\right]-1\right)-R\left(\sum_{i=1}^{N} \bar{\mu}_{i}\left(\bar{t}, \bar{X}^{\dagger}\right)\right) .
\end{aligned}
$$

We also have that 
$\mathbf{E}\left[f_{\bar{t}+1}^{\bar{\mu}}\left(\bar{X}^{+1+1}\right) \mid \overline{X^{t}}, \bar{\mu}\left(\bar{t}, \bar{X}^{\dagger}\right)\right]$

$$
\begin{aligned}
& -\mathbf{E}\left[f_{\bar{t}+1}^{\bar{\mu}}\left(\bar{X}^{\overline{+1}}\right) \mid \bar{X}^{t}, 1,1, \bar{\mu}_{3}(\bar{t}, \bar{X}), \ldots, \bar{\mu}_{N}(\bar{t}, \bar{X})\right]=\mathbf{E}_{\mathbf{X}_{3}^{+1}, \ldots, \overline{\mathbf{X}}_{\mathrm{N}}^{++1}}^{\overline{+1}}\left[\sum_{j_{2} \in \mathscr{S}} p_{0 j_{2}} \cdot \sum_{j_{1} \in \mathscr{S}}\left(p_{k j_{1}}-p_{0 j_{1}}\right)\right. \\
& \left.\cdot f_{\bar{t}+1}^{\bar{\mu}}\left(j_{1}, j_{2}, \bar{X}_{3}^{i+1}, \ldots, \bar{X}_{N}^{\bar{t}+1}\right) \mid \bar{X}_{3}^{t}, \ldots, \bar{X}_{N}^{t}, \bar{\mu}_{3}\left(\bar{t}, \bar{X}^{\bar{\gamma}}\right), \ldots, \bar{\mu}_{N}\left(\bar{t}, \bar{X}^{\dagger}\right)\right] \text {. }
\end{aligned}
$$

Since $\sum_{j_{1} \in \mathscr{S}}\left(p_{k j_{1}}-p_{0 j_{1}}\right) \cdot f_{t+1}^{\bar{\mu}}\left(j_{1}, j_{2}, X_{3}^{\bar{t}+1}, \ldots, X_{N}^{\bar{t}+1}\right)$ is nondecreasing in $j_{2}$, Derman's Lemma implies that: $\sum_{j_{2} \in \mathscr{S}}\left(p_{k j_{2}}-p_{0 j_{2}}\right) \sum_{j_{1} \in \mathscr{S}}\left(p_{k j_{1}}-p_{0 j_{1}}\right) \cdot f_{t+1}^{\bar{u}}\left(j_{1}, j_{2}\right.$, $\left.X_{3}^{t+1}, \ldots, X_{N}^{\bar{t}+1}\right) \geq 0$. In turn, this means that

$$
\begin{aligned}
& \mathbf{E}\left[f_{\bar{t}+1}^{\bar{\mu}}\left(\bar{X}^{\overline{t+1}}\right) \mid \bar{X}^{t}, \bar{\mu}\left(\bar{t}, \bar{X}^{\bar{X}}\right)\right] \\
& -\mathbf{E}\left[f_{\bar{t}+1}^{\bar{\mu}}\left(\bar{X}^{\bar{t}+1}\right) \mid \bar{X}^{t}, 1,1, \bar{\mu}_{3}\left(\bar{t}, \bar{X}^{\bar{\phi}}\right), \ldots, \bar{\mu}_{N}\left(\bar{t}, \bar{X}^{\bar{X}}\right)\right] \\
& \geq \mathbf{E}\left[f_{t+1}^{\bar{\mu}}\left(\bar{X}^{i+1}\right) \mid \bar{X}^{t}, 0,0, \bar{\mu}_{3}\left(\bar{t}, \bar{X}^{\bar{\eta}}\right), \ldots, \bar{\mu}_{N}\left(\bar{t}, \bar{X}^{\bar{\dagger}}\right)\right] \\
& -\mathbf{E}\left[f_{\bar{t}+1}^{\bar{\mu}}\left(\bar{X}^{\overline{t+1}}\right) \mid \bar{X}^{t}, \bar{\mu}\left(\bar{t}, \bar{X}^{\bar{t}}\right)\right] .
\end{aligned}
$$

Together, Eqs. (14) and (15) lead to the conclusion that there is nonnegative incremental value in replacing both machines for $\bar{t}$ and $\bar{X}^{\bar{t}}$, i.e., Eq. (13) is greater than or equal to the left-hand side of Eq. (12), which is nonnegative. By applying the argument repeatedly, it is possible to construct a policy that satisfies the NSR and that is at least as good as $\bar{\mu}$. This contradicts the assumption that there is no optimal policy for the problem instance that satisfies the NSR, and therefore, completes the proof.

The proof of the NSR presented in [7] for the deterministic PMRP relies on fixed plus variable replacement costs. Above, we extended the result to the general class of functions with nonincreasing marginal costs. The PMRP literature incorrectly suggests that the NSR derives from economies of scale in replacement costs. The following example provides an instance of the deterministic PMRP with economies of scale in replacement costs where the NSR does not hold. Prior to presenting the example and to clarify the distinctions between different types of functions, we present definitions for functions that exhibit economies of scale. We adapt these definitions from Varian [11]:

DEFINITION 2: A replacement cost function exhibits economies of scale if

$$
R(c y) \leq c R(y), \quad \forall c \geq 1, \quad y \geq 0 .
$$

DEFINITION 3: A replacement cost function has nonincreasing average costs if

$$
\frac{R(y+\Delta)}{y+\Delta} \leq \frac{R(y)}{y}, \quad \forall \Delta \geq 0, \quad y \geq 0 .
$$

For $y=0$, the average replacement costs are defined as $\lim _{y \rightarrow 0^{+}}[R(y) / y]$.

Replacement cost functions that have nonincreasing marginal costs (i.e., that satisfy Definition 1) exhibit economies of scale. The replacement cost function used in the following example shows that the reverse is not true in general.

EXAMPLE 1: We present a finite horizon (1 stage) instance of the deterministic PMRP with economies of scale in replacement costs where the NSR does not hold. In this example, we let the replacement cost function be

$$
R(y)= \begin{cases}0, & y=0 \\ 1, & 0<y \leq 2, \\ \frac{1}{2} y, & y \geq 2\end{cases}
$$

As illustrated in Figure 1, the replacement cost function given in Eq. (18) has nonincreasing average costs per unit and hence exhibits economies of scale. However, the function does not exhibit nonincreasing marginal costs; i.e., it does not satisfy Definition 1 . For example, if we let $y_{1}=1$, $y_{2}=2, \Delta=1$, then $R\left(y_{2}+\Delta\right)-R\left(y_{2}\right)>R\left(y_{1}+\Delta\right)-$ $R\left(y_{1}\right)$.

Next, we construct an instance of the deterministic PMRP using the replacement cost function given by (18) such that the NSR does not hold. Consider a population of 3 machines of ages 1,1 , and 2 , and suppose that the salvage value for machines of all ages is $0[s(i)=0, \forall i]$, and that the maintenance and operating costs are given by the function $m(i)=i^{2} / 3, \forall i$. The policy to replace the machine of age 2 and one of the machines of age 1 , thus splitting the cluster of age 1 , is optimal with a cost of $4 / 3$. The cost of this policy is strictly less than the cost associated with implementing any other policy. This counterexample demonstrates that replacement cost functions that exhibit economies of scale do not guarantee the existence optimal replacement policies satisfying the NSR.

Example 1 and Theorem 3 reveal that optimal replacement decisions for the PMRP are driven by the marginal 


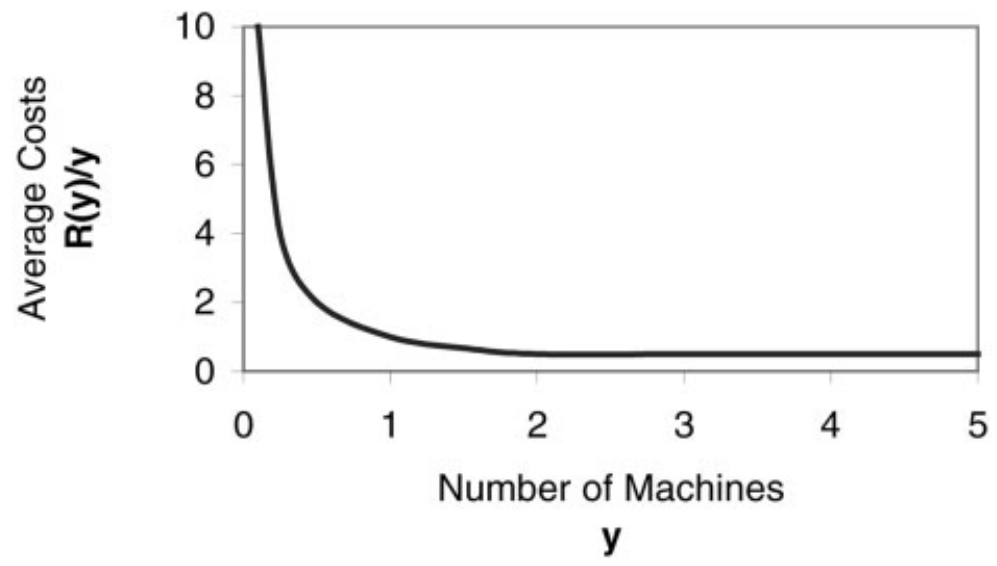

Figure 1. Average replacement costs per unit.

cost structure and not by economies of scale. That is, decisions to replace additional machines are evaluated based on their incremental value (marginal benefits minus marginal costs). Nonincreasing marginal replacement costs guarantee that there is (nonnegative) value in replacing additional machines of the same age, whereas, nonincreasing average replacement costs per unit do not. The results in the literature are obtained for replacement cost functions consisting of fixed and per-unit variable costs. These cost functions satisfy Definition 1 (nonincreasing marginal costs), and therefore, are members of a special class of functions that satisfy Definition 2 (economies of scale).

\section{FINDING OPTIMAL REPLACEMENT POLICIES}

Finding an optimal replacement policy for the PMRP involves selecting a best replacement option for every possible state $\vec{X}^{t}$ and stage $t$, i.e., finding $\mu^{*}\left(t, \vec{X}_{t}\right), \forall \vec{X}_{t} \in \mathscr{S}^{N}$, $t=1, \ldots, T$. Computational approaches for the problem usually involve both the enumeration of each replacement option for every stage and state combination (an exponential number), as well as, the solution of an optimization problem. In this section, we consider a linear programming approach to find optimal replacement policies for the infinite-horizon, ${ }^{2}$ stochastic PMRP with stationary parameters. Through the analysis of the linear program (LP), we illustrate how the NSR and WCRR reduce the computational effort required to find optimal replacement policies for the stochastic PMRP. We begin by discussing the existing computational approaches for the deterministic PMRP with fixed plus variable replacement costs.

\footnotetext{
${ }^{2}$ Since the structural results described in the previous section apply to arbitrary planning horizons, they also apply to the infinitehorizon problem.
}

\subsection{Solving the Deterministic PMRP}

As discussed in Section 2, Jones, Zydiak, and Hopp [7] formulate the deterministic PMRP as a (backward) dynamic program. They consider infinite-horizon problems with stationary parameters and propose a solution strategy that requires solving a LP. It is possible to develop a similar LP for finite-horizon problems with time-varying parameters; however, optimal policies for finite-horizon dynamic programs are usually computed recursively by backwards induction. For the infinite-horizon problem with time-varying parameters, Jones, Zydiak, and Hopp [7] explain that by invoking standard forecast horizon results, optimal decisions for a given number of periods can be obtained by solving a sequence of finite-horizon problems in a rollinghorizon fashion. In all cases, the size of the underlying optimization problem, and hence the computational effort to obtain optimal replacement policies, grows exponentially with the number of initial clusters, $n$. McClurg and Chand [9] introduce a forward dynamic program that relies on the OCRR and on a dominance property for finite-horizon problems. This approach is slightly more efficient than that of [7] because it is only necessary to specify replacement options for the stage and state combinations that are consistent with the ages of the initial clusters that comprise the population. Unfortunately, the size of the optimization problem grows exponentially with the number of stages, $T$. Finally, Chen [2] proposes shortest path algorithms that exploit the AONR for infinite-horizon problems with stationary parameters and for finite-horizon problems with time-varying parameters. These algorithms are efficient but rely on overly restrictive assumptions to ensure that the AONR applies, as discussed earlier.

In addition to the aforementioned algorithms, Chen [2] formulates the finite horizon PMRP with fixed plus variable replacement costs and time-varying parameters as a zeroone integer program. He proposes an exact solution proce- 
dure based on Benders' decomposition. The key to the efficiency of this approach is that the decision to allow replacements in a given stage is separated from the choice of which clusters to replace. After the selection of a given set of stages in which replacements are allowed, the problem can be decomposed into $n$ subproblems, one for each cluster. The subproblems are independent which means that the replacement decisions for each of the clusters does not depend on the ages of the other clusters, and therefore, it is not necessary to list the exponential number of possible stage-state combinations in the original problem. Unfortunately, the selection of an optimal set of stages in which replacements are allowed, involves solving a sequence of zero-one integer programs. Furthermore, the number of binary variables in the integer programs increases linearly with the length of the planning horizon, $T$. Through a computational study, however, the author shows that the procedure is capable of solving large-scale problems, and that the computational time increases slowly with $T$.

Except for the zero-one integer program of [2] that relies on the fixed plus variable cost structure, the computational approaches described above can be used to find optimal replacement policies for deterministic problems with general classes of replacement cost functions. ${ }^{3}$ That is, the linear programming approach of [7] or the forward dynamic programming approach of [9] can be used for the deterministic PMRP with nonincreasing marginal costs because the NSR applies. The OCRR is also required for the forward dynamic programming approach of [9]. Also, one may use the shortest path algorithms of [2] if the assumptions required for the AONR hold. These algorithms, however, cannot be used to solve the stochastic PMRP because they assume that the number of clusters in the population does not increase over the planning horizon (a corollary to the NSR). In the stochastic PMRP, clusters can be split even if the same actions are applied to them. In addition, the forward dynamic programming formulation of [9] and the zero-one integer program of [2] rely on the fact that machines deteriorate deterministically.

\subsection{Solving the Stochastic PMRP}

Next, we consider a linear programming approach to find optimal replacement policies for the infinite-horizon, sto-

\footnotetext{
${ }^{3}$ The algorithm of [2] does not extend to the case of general replacement costs because it is not possible to separate the clusters that comprise the population. With a general replacement cost structure, after the decision to allow replacements in a given stage, the marginal replacement costs for a given cluster can still depend on the decisions for the other clusters in the population.
}

chastic PMRP with stationary parameters. ${ }^{4}$ The insights we develop, however, generalize to problems with finite horizons or time-varying parameters. The computational effort required to solve the infinite-horizon, stochastic PMRP with stationary parameters relates to the fact that the size of the corresponding LP grows exponentially with the parameters of the problem. For this reason, our discussion of complexity focuses on the size of the LP. The number of variables and constraints in the LP are $(\bar{a}+1)^{N}$ and $2^{N} \cdot(\bar{a}+1)^{N}$, respectively. The number of variables comes from the number of possible state-vectors used to describe the population. Each machine can be in one of $(\bar{a}+1)$ states, and therefore, the total number of states is $(\bar{a}+1)^{N}$. The number of constraints is equal to the product of the number of states times the number of replacement options available for each state. As each machine may either be kept or replaced, the number of replacement options for each state is $2^{N}$.

Because the machines in the population are homogeneous with respect to costs and deterioration, it is possible to relabel them so that their states are ordered from best to worst. Jones, Zydiak, and Hopp [7] show that this reduces the number of variables in the linear program to $\mathscr{C}_{N}^{N+\bar{a}}$, i.e., the number of combinations of size $N$ from a set of size $N+$ $\bar{a}$. They also make use of the facts (i) that there is an optimal policy where machines in state 0 are kept, (ii) that machines in state $\bar{a}$ must be replaced, and (iii) that for two machines in the same state it is equivalent to replace one or the other, to show that the number of constraints in the LP reduces to $\sum_{k=0}^{N} \mathscr{C}_{N-k}^{N-k+1} \cdot \mathscr{C}_{k}^{\bar{a}-2+k} \cdot 2^{k}=\sum_{k=0}^{N}(N-k+1)$. $\mathscr{C}_{k}^{\bar{a}-2+k} \cdot 2^{k}$.

We now show how the WCRR and the NSR reduce the number of constraints in the LP, and thus the computational effort to solve the infinite-horizon, stochastic PMRP with stationary parameters. Recall that the number of constraints corresponds to the total number of replacement options. If the WCRR holds, however, at most $N+1$ replacement options need to be considered for each state, instead of at most $2^{N}$. To clarify the number or replacement options when the WCRR holds, consider a population of three machines. The possible replacement options are to either do nothing, or to replace machine 3 (the one in the worst state), or to replace machines 2 and 3 (the two worst machines), or to replace the three machines. The total number of constraints in the LP in general when the WCRR applies is $\sum_{k=0}^{N}(N-k+1) \cdot \mathscr{C}_{k}^{\bar{a}-2+k} \cdot(k+1)$.

When the NSR applies, there is an optimal replacement policy that prescribes the same decision for all of the machines in the same state. In the three-machine example, in states where all machines are the same age, the possible

\footnotetext{
${ }^{4}$ References such as Bertsekas [1] and Feinberg and Shwartz [4] provide background on using LPs to solve stochastic dynamic programs.
} 
Table 1. LP size to solve the infinite-horizon PMRP with stationary parameters.

\begin{tabular}{lcrrrr}
\hline & \multicolumn{2}{c}{ Deterministic problem } & & \multicolumn{2}{c}{ Stochastic problem } \\
\cline { 2 - 3 } Applicable rules & Variables & Constraints & & Variables & Constraints \\
\hline None & 15,504 & $77,070,360$ & & 15,504 & $77,070,360$ \\
OCRR/WCRR only & 15,504 & 170,544 & & 15,504 & 170,544 \\
NSR only & 360 & 2496 & & 15,504 & 246,048 \\
NSR \& OCRR/WCRR & 360 & 1320 & & 15,504 & 73,646 \\
\hline
\end{tabular}

options are to either do nothing or to replace the three machines. The NSR produces significant reductions in the number of constraints in the LP when the size of the population, $N$, is large relative to the number of states, $\bar{a}+$ 1. In the special case of the deterministic PMRP, where it is possible to redefine the state-space of the problem to be the sizes and ages of the clusters that comprise the population, the number of variables in the LP reduces to $P_{n}^{\bar{a}+1}$, i.e., the number of permutations of size $n$ from a set of size $\bar{a}+1$. The number of constraints in the LP is $2^{n} \mathscr{P}_{n}^{\bar{a}-1}+2^{n} \mathscr{P}_{1}^{n}$. $\mathscr{P}_{n-1}^{\bar{a}-1}+2^{n-2} \mathscr{P}_{2}^{n} \cdot \mathscr{P}_{n-2}^{\bar{a}-1}$, when the OCRR does not apply, and $(n+1) \mathscr{P}_{n}^{\bar{a}-1}+2 n \mathscr{P}_{1}^{n} \cdot \mathscr{P}_{n-1}^{\bar{a}-1}+(n-1) \mathscr{P}_{2}^{n} \cdot \mathscr{P}_{n-2}^{\bar{a}-1}$, when it does.

To make the above discussion tangible, we consider the following example:

EXAMPLE 2: We consider infinite-horizon, deterministic and stochastic instances of the PMRP with stationary parameters. These instances involve 15 machines initially clustered in 4 different ages/states, with the maximum allowable age/state of 5, i.e., $N=15, n=4$, and $\bar{a}=5$. The number of variables and constraints in the LP, depending on the rules that apply, are shown in Table 1.

The example illustrates how making use of the structural results can significantly reduce the size of the optimization problem, and hence the computational effort, to solve the PMRP. Interestingly, we observe that the OCRR/WCRR alone can significantly reduce the number of replacement options that need to be considered. This result can be particularly useful for PMRPs with arbitrary replacement cost functions or with stochastic deterioration. This case is not considered in the literature because earlier proofs of the OCRR assume that the NSR, which requires a replacement cost function with nonincreasing marginal costs, holds. Also, as expected, we observe that the NSR can substantially reduce the size of deterministic PMRPs because it is only necessary to keep track of clusters, as opposed to individual machines, in the state-space of the problem.

\section{SUMMARY AND CONCLUSIONS}

The existing literature on the PMRP centers on two properties for the case of deterministic deterioration and replacement cost functions consisting of fixed and per-unit variable purchase costs. The NSR ensures that there is an optimal replacement policy such that in any stage machines of the same age are either all kept or all replaced. The OCRR guarantees that there is an optimal policy such that a machine is replaced only if all older machines are replaced.

In this paper, we formulate the stochastic PMRP as a set of independent Markovian processes and show that under the assumption of an increasing failure rate the structure of optimal replacement policies for the deterministic PMRP extends to the stochastic version of the problem. Specifically, we prove that optimal policies for the stochastic PMRP with arbitrary replacement cost functions satisfy a result analogous to the OCRR. We also extend the NSR for replacement functions with nonincreasing marginal costs. In addition, we present an example that shows that economies of scale in replacement costs do not guarantee optimal replacement policies that satisfy the NSR. Together, the example and the results lead to the conclusion that replacement decisions are driven by marginal costs, and not by economies of scale as suggested in the PMRP literature.

Finally, we consider a linear programming formulation to find optimal replacement policies for the infinite-horizon stochastic PMRP with stationary parameters. Through the analysis of the linear program, we illustrate how the NSR and WCRR reduce the computational effort required to solve the stochastic PMRP.

\section{APPENDIX: PROOFS}

LEMMA 1: Under assumptions (a) and (b), the optimal objective value function of the stochastic PMRP with increasing failure rate is nondecreasing in each component of the state-space, i.e.,

$$
\begin{aligned}
f_{t}^{\mu^{*}}\left(i, X_{2}^{t}, \ldots, X_{N}^{t}\right) & \leq f_{t}^{\mu^{*}}\left(j, X_{2}^{t}, \ldots, X_{N}^{t}\right), \quad \forall i \\
\leq j & \in \mathscr{S}, \quad X_{2}^{t}, \ldots, X_{N}^{t} \in \mathscr{Y}^{N-1}, \quad t=1,2, \ldots, T+1 .
\end{aligned}
$$

PROOF: The proof is by induction. Equation (6) and assumption (b) establish the result for stage $T+1$. We assume that the result holds for stage $t+1$ and consider stage $t$. We compare the expected discounted costs through the end of the horizon when the set of actions $\mu^{*}(t, j$, $X_{2}^{t}, \ldots, X_{N}^{t}$ ) is applied to both states in stage $t$, and policy $\mu^{*}$ is followed thereafter. We have two cases:

$$
\begin{gathered}
\mu_{1}^{*}\left(t, j, X_{2}^{t}, \ldots, X_{N}^{t}\right)=1: \text { In this case, } \\
f_{t}^{\mu^{*}}\left(j, X_{2}^{t}, \ldots, X_{N}^{t}\right)-\bar{f}_{t}\left(i, X_{2}^{t}, \ldots, X_{N}^{t}\right)=s(i)-s(j) \geq 0
\end{gathered}
$$

by assumption (b). 
In this case, $\bar{f}_{t}\left(i, X_{2}^{t}, \ldots, X_{N}^{t}\right)$ represents the total expected discounted cost (until the end of the horizon) of applying the set of actions $\mu^{*}(t, j$, $X_{2}^{t}, \ldots, X_{N}^{t}$ ) in stage $t$ to the system in state $i, X_{2}^{t}, \ldots, X_{N}^{t}$ and following policy $\mu^{*}$ thereafter.

$$
\begin{aligned}
& \mu_{1}^{*}\left(t, j, X_{2}^{t}, \ldots, X_{N}^{t}\right)=0 \text { : In this case, } \\
& f_{t}^{\mu^{*}}\left(j, X_{2}^{t}, \ldots, X_{N}^{t}\right)-\hat{f}_{t}\left(i, X_{2}^{t}, \ldots, X_{N}^{t}\right)=m(j)-m(i) \\
& +\delta \cdot\left\{\begin{array}{l}
\mathbf{E}\left[f_{t+1}^{\mu^{*}}\left(\vec{X}^{t+1} \mid j, X_{2}^{t}, \ldots, X_{N}^{t}, \mu^{*}\left(t, j, X_{2}^{t}, \ldots, X_{N}^{t}\right)\right)\right] \\
-\mathbf{E}\left[f_{t+1}^{\mu^{*}}\left(\vec{X}^{t+1} \mid i, X_{2}^{t}, \ldots, X_{N}^{t}, \mu^{*}\left(t, j, X_{2}^{t}, \ldots, X_{N}^{t}\right)\right)\right]
\end{array}\right\} .
\end{aligned}
$$

In this case, $\hat{f}_{t}\left(i, X_{2}^{t}, \ldots, X_{N}^{t}\right)$ represents the total expected discounted cost (until the end of the horizon) of applying the set of actions $\mu^{*}(t, j$, $\left.X_{2}^{t}, \ldots, X_{N}^{t}\right)$ in stage $t$ to the system in state $i, X_{2}^{t}, \ldots, X_{N}^{t}$ and following policy $\mu^{*}$ thereafter. We have that $m(j)-m(i) \geq 0$ by assumption (a). We evaluate the expectations by conditioning on the realizations of $X_{2}^{t+1}, \ldots, X_{N}^{t+1}$, i.e.,

$$
\begin{aligned}
& \mathbf{E}\left[f_{t+1}^{\mu}\left(\vec{X}^{t+1} \mid \vec{X}^{t}, \mu\left(t, \vec{X}^{t}\right)\right)\right] \\
& \quad=\mathbf{E}_{\mathbf{X}_{2}^{t+1}, \ldots, \mathbf{X}_{N}^{t+1}}\left[\mathbf{E}_{\mathbf{X}_{1}^{t+1}} \mid \mathbf{X}_{2}^{t+1}, \ldots, \mathbf{X}_{N}^{t+1}\left[f_{t+1}^{\mu}\left(\vec{X}^{t+1} \mid \vec{X}^{t}, \mu\left(t, \vec{X}^{t}\right), X_{2}^{t+1}, \ldots, X_{N}^{t+1}\right)\right]\right] .
\end{aligned}
$$

Since the deterioration process of each machine is independent, the conditional expectation of $X_{1}^{t+1}$ given the realizations of $X_{2}^{t+1}, \ldots, X_{N}^{t+1}$ is equal to its (unconditional) expectation. Therefore, the last expression may be written as

$$
\mathbf{E}_{\mathbf{X}_{2}^{t+1}, \ldots, \mathbf{X}_{\mathrm{N}}^{t+1}}\left[\sum_{k \in \mathscr{S}} p_{X_{1}^{t} k} \cdot f_{t+1}^{\mu}\left(k, X_{2}^{t+1}, \ldots, X_{N}^{t+1} \mid \vec{X}^{t}, \mu\left(t, \vec{X}^{t}\right), X_{2}^{t+1}, \ldots, X_{N}^{t+1}\right)\right] .
$$

The induction hypothesis and Derman's Lemma imply that

$$
\begin{aligned}
& \sum_{k \in \mathscr{S}} p_{i k} \cdot f_{t+1}^{u^{*}}\left(k, X_{2}^{t+1}, \ldots, X_{N}^{t+1} \mid i,\right. \\
& \left.X_{2}^{t}, \ldots, X_{N}^{t}, \mu^{*}\left(t, j, X_{2}^{t}, \ldots, X_{N}^{t}\right), X_{2}^{t+1}, \ldots, X_{N}^{t+1}\right) \\
& \quad \leq \sum_{k \in \mathscr{S}} p_{j k} \cdot f_{t+1}^{\mu^{*}}\left(k, X_{2}^{t+1}, \ldots, X_{N}^{t+1} \mid j,\right. \\
& \left.X_{2}^{t}, \ldots, X_{N}^{t}, \mu^{*}\left(t, j, X_{2}^{t}, \ldots, X_{N}^{t}\right), X_{2}^{t+1}, \ldots, X_{N}^{t+1}\right), \quad \forall i \leq j \in \mathscr{Y} .
\end{aligned}
$$

We use the last expression to obtain the inequality $\mathbf{E}\left[f_{t+1}^{u^{*}}\left(\vec{X}^{t+1} \mid j\right.\right.$, $\left.\left.X_{2}^{t}, \ldots, X_{N}^{t}, \mu^{*}\left(t, j, X_{2}^{t}, \ldots, X_{N}^{t}\right)\right)\right]-\mathbf{E}\left[f_{t+1}^{\mu *}\left(\vec{X}^{t+1} \mid i, X_{2}^{t}, \ldots, X_{N}^{t}\right.\right.$, $\left.\left.\mu^{*}\left(t, j, X_{2}^{t}, \ldots, X_{N}^{t}\right)\right)\right] \geq 0$. From this we conclude that when $\mu_{1}^{*}(t, j$, $\left.X_{2}^{t}, \ldots, X_{N}^{t}\right)=0, f_{t}^{\mu^{*}}\left(j, X_{2}^{t}, \ldots, X_{N}^{t}\right)-\hat{f}_{t}\left(i, X_{2}^{t}, \ldots, X_{N}^{t}\right) \geq 0$. By definition, $\mu^{*}\left(t, i, X_{2}^{t}, \ldots, X_{N}^{t}\right)$ is at least as good as any set of actions that can be applied in stage $t$ to a system in state $i, X_{2}^{t}, \ldots, X_{N}^{t}$.

Depending on the case that applies, $f_{t}^{\mu^{*}}\left(i, X_{2}^{t}, \ldots, X_{N}^{t}\right)$ is less than or equal to either $\bar{f}_{t}\left(i, X_{2}^{t}, \ldots, X_{N}^{t}\right)$ or $\hat{f}_{t}\left(i, X_{2}^{t}, \ldots, X_{N}^{t}\right)$, which in turn completes the proof.

\section{REFERENCES}

[1] D. Bertsekas, Dynamic programming and optimal control, Athena Scientific, Belmont, MA, 2000.

[2] Z. Chen, Solution algorithms for the parallel replacement problem under economy of scale, Naval Res Logist 45 (1998), 279-295.

[3] C. Derman, Mathematical optimization techniques, University of California Press, Berkeley, 1963.

[4] E. Feinberg and A. Shwartz, Handbook of Markov decision processes, Kluwer Academic, Norwell, MA, 2002.

[5] J.C. Hartman, A general procedure for incorporating asset utilization into replacement analysis, Eng Econom 44(3) (1999), 217-238.

[6] W.J. Hopp, P.C. Jones, and J.L. Zydiak, A further note on parallel machine replacement, Naval Res Logist 40 (1993), 575-579.

[7] P.C. Jones, J.L. Zydiak, and W.J. Hopp, Parallel machine replacement, Naval Res Logist 38 (1991), 351-365.

[8] N. Karabakal, J. Lohmann, and J. Bean, Parallel replacement under capital rationing constraints, Management Sci 40(3) (1994), 305-319.

[9] T. McClurg and S. Chand, A parallel machine replacement model, Naval Res Logist 49 (2002), 275-287.

[10] J. Tang and K. Tang, A note on parallel machine replacement, Naval Res Logist 40 (1993), 569-573.

[11] H. Varian, Microeconomic analysis, Norton, New York, 1992.

[12] D.J. Vander Veen, Parallel replacement under nonstationary deterministic demand, Ph.D. thesis, University of Michigan, Ann Arbor, 1985. 